\title{
Formation and dynamics of fission fragments
}

\author{
C. Simenel ${ }^{1}$ and A. S. Umar ${ }^{2}$ \\ ${ }^{1}$ Department of Nuclear Physics, Research School of Physics and Engineering, Australian National University, \\ Canberra, Australian Capital Territory 0200, Australia \\ ${ }^{2}$ Department of Physics and Astronomy, Vanderbilt University, Nashville, Tennessee 37235, USA \\ (Received 17 December 2013; revised manuscript received 21 January 2014; published 21 March 2014)
}

\begin{abstract}
Although the overall time scale for nuclear fission is long, suggesting a slow process, rapid shape evolution occurs in its later stages near scission. Theoretical prediction of the fission fragments and their characteristics are often based on the assumption that the internal degrees of freedom are equilibrated along the fission path. However, this adiabatic approximation may break down near scission. This is studied for the symmetric fission of ${ }^{258,264} \mathrm{Fm}$. The nonadiabatic evolution is computed using the time-dependent Hartree-Fock method, starting from an adiabatic configuration where the fragments have acquired their identity. It is shown that dynamics has an important effect on the kinetic and excitation energies of the fragments. The vibrational modes of the fragments in the post-scission evolution are also analyzed.
\end{abstract}

DOI: 10.1103/PhysRevC.89.031601

PACS number(s): 24.75.+i, 21.60.Jz

Fission can be found in different complex quantum systems such as atomic nuclei [1,2] and atomic clusters [3]. This is one of the most challenging quantum many-body problems, due to the difficulty of finding an adequate and computationally tractable formulation of the evolution from the compound system to the formation of the final fragments. For atomic nuclei, the minimum average time scale for such an evolution is of the order of 20-50 zs [4]. This is slow enough to consider, as a first approximation, nuclear fission as an adiabatic process. This means that the nucleonic degrees of freedom are fully equilibrated while the system evolves over a potential energy surface (PES) defined by the macroscopic degrees of freedom such as elongation and mass asymmetry [5].

However, the adiabatic approximation is expected to break down in the final stages of the fission process, when scission of the neck between the fragments occurs [6]. In this phase, fragments can experience a rapid change in shape [7], which would be better described with a nonadiabatic approach. A realistic description of the entire fission process could then be achieved with an adiabatic model describing the slow evolution across the barrier, followed by a nonadiabatic treatment of the scission and post-scission dynamics. The transition between the adiabatic and nonadiabatic pictures is expected to occur somewhere between the top of the fission barrier and the scission point. It is desirable that these two methods are based on a consistent approach to the many-body problem.

To date, most of the theoretical works have focused on the adiabatic part of the fission process. Microscopic approaches have been widely used to study fission paths (see Refs. [7-20] for recent applications). In particular, the time-dependent generator coordinate method [8] and the adiabatic timedependent Hartree-Fock theory [21] provide a description of the evolution of collective and internal degrees of freedom. Simplifications using the strongly damped character of fission have also been widely used. For instance, random walks on a phenomenological five-dimensional PES [22], in analogy with Brownian motion, have led to a good description of fragment mass distributions [23].
All of these approaches aim to describe the fission process up to the scission point and to predict the properties of the fragments (mass, charge, kinetic energy), which are the main experimental observables. These properties are estimated in a sudden approximation from the scission configuration assuming sharp cuts across the neck [24] and ignoring nonadiabatic effects coming from couplings between collective and intrinsic degrees of freedom [6,25]. This approximation induces a strong limitation in the predicting power of fragment characteristics. For instance, part of the kinetic energy could come from pre-scission dynamics [12]. In addition, the approach is not able to describe post-scission dynamics of the fragments, such as their vibrational modes. For this reason, it is highly desirable to go beyond this approximation and describe the later stages of the fission process in a dynamical and nonadiabatic fashion [26]. The time-dependent Hartree-Fock (TDHF) theory [27] is an ideal tool to study the latter stage of fission as it is a fully microscopic and nonadiabatic approach. An early attempt to describe fission with such a model was proposed in Ref. [28]. Due to computational limitations, these calculations were essentially qualitative, assuming spatial symmetries and using a simplified effective interaction. A pairing gap with arbitrarily large values was also used as a phenomenological parameter to trigger scission.

Here, we investigate the formation and dynamics of fission fragments using a realistic three-dimensional meanfield description. The adiabatic phase is described in the traditional way, using a static mean-field approach with an external constraint inducing deformation. The shell structure and level crossings are used to determine at which deformation the fragments have established their identity, which occurs between the saddle and scission points [29]. This determines the initial condition for the time-dependent calculations of the nonadiabatic evolution, including scission and post-scission dynamics.

As a proof of concept and to establish the feasibility of this approach we studied the symmetric fission of ${ }^{264} \mathrm{Fm}$. This exotic nucleus represents an important milestone in fission studies as it is predicted to spontaneously fission into two 


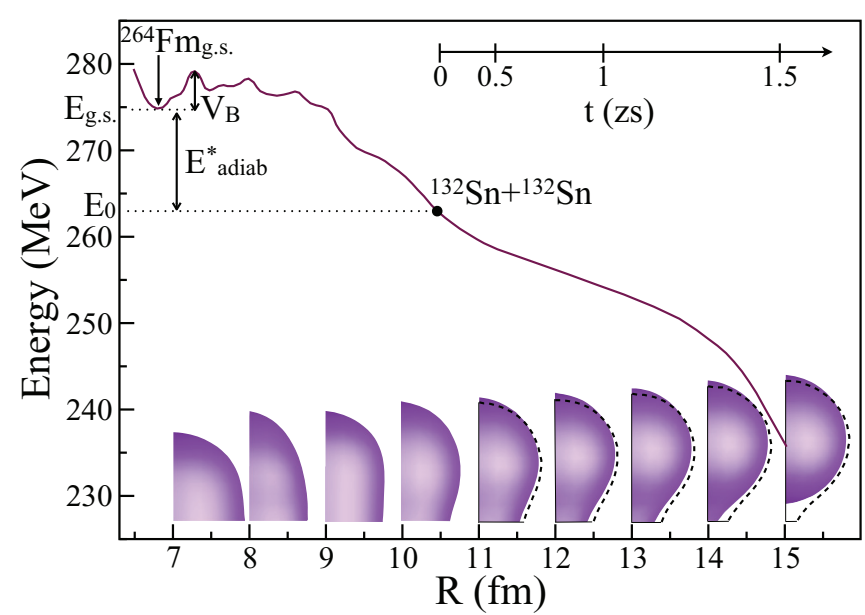

FIG. 1. (Color online) Adiabatic fission potential of ${ }^{264} \mathrm{Fm} \rightarrow$ ${ }^{132} \mathrm{Sn}+{ }^{132} \mathrm{Sn}$ (solid line) as function of the distance $R$ between the fragment centers of mass. The reference energy is chosen such that $E=0$ for $R \rightarrow \infty$. Adiabatic (purple surfaces) isodensities at half the saturation density $\rho_{0} / 2=0.08 \mathrm{fm}^{-3}$ are shown at $R=$ $7,8, \ldots, 15 \mathrm{fm}$. Half a fragment is represented, the fission axis being vertical. TDHF isodensities are represented by dashed lines at $R=11,12, \ldots, 15 \mathrm{fm}$. The nonlinear axis (top) relates the time $t$ with $R$ during the nonadiabatic evolution, with origin $t=0$ associated with preformation of ${ }^{132} \mathrm{Sn}$ fragments.

doubly magic ${ }^{132} \mathrm{Sn}$ fragments [18,30,31]. Its study could be envisaged with upcoming radioactive beams or, alternatively, using multi-nucleon transfer reactions in actinide collisions, which have been the focus of recent experimental [32] and theoretical [33-35] efforts.

The adiabatic configurations are obtained by solving the Hartree-Fock (HF) equations with the BCS pairing residual interaction $(\mathrm{HF}+\mathrm{BCS})$ using the EV8 code [36]. The calculations are performed on a Cartesian grid with mesh size $0.8 \mathrm{fm}$. The mean field is obtained from the SLy4d [37] Skyrme energy density functional [38], and a surface pairing interaction [39] is used to describe the nuclear superfluid phase. Elongations along the $z$ axis are induced by adding an external constraint $\lambda(\langle\hat{R}\rangle-R) z$ to the single-particle potential. The Lagrange parameter $\lambda$ quantifies the strength of the constraint and $R$ is the desired expectation value of the operator $\hat{R}$ measuring the distance between the centers of mass of the matter on each side of the neck plane assuming a sharp cut. For symmetric fission, the neck is at $z=0$.

The adiabatic potential obtained from the constraint calculations is shown in Fig. 1. The fission barrier height is $V_{B} \simeq$ $4.3 \mathrm{MeV}$ at $R_{B} \simeq 7.3 \mathrm{fm}$. This height is in excellent agreement with recent theoretical calculations $[10,11,18,40,41]$. A neck is observed up to $R \simeq 14.5 \mathrm{fm}$. It is interesting to note that the pre-scission configuration consists of two quasi-spherical fragments. In fact, three different fission valleys have been predicted in fermium isotopes [9,31]: (i) a mass asymmetric one, (ii) a mass symmetric one with elongated fragments, and (iii) a mass symmetric one with compact fragments like the one in Fig. 1. The latter is the dominant fission path in neutron-rich fermium isotopes due to the spherical shells in the vicinity of ${ }^{132} \mathrm{Sn}[42,43]$.
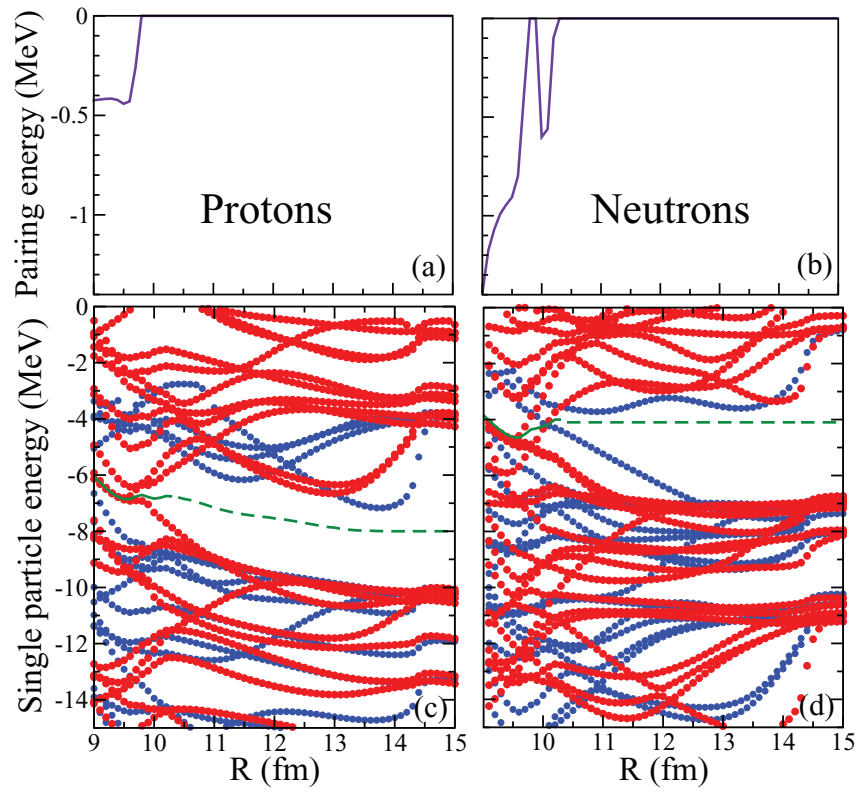

FIG. 2. (Color online) Proton (a) and neutron (b) pairing energies as functions of $R$. Proton (c) and neutron (d) single-particle energies for states with positive (blue) and negative (red) parity. The green solid lines indicate the Fermi levels in the presence of pairing. These are continued by green dashed lines, which represent the Fermi levels located arbitrarily in the magic gaps.

The transition criteria between adiabatic and nonadiabatic phases has yet to be defined. A distance $R_{0}$ where fragments are pre-formed can be determined by examining the shell structure of the system. The proton and neutron single-particle energies are plotted near the Fermi level for $R>9 \mathrm{fm}$ in Figs. 2(c) and 2(d), respectively. We observe that the shell gaps, $Z=50$ and $N=82$, associated with ${ }^{132} \mathrm{Sn}$ appear after $R \approx 10 \mathrm{fm}$. This is also confirmed by the evolution of the proton and neutron pairing energies shown in Figs. 2(a) and 2(b), respectively. The latter vanish around the same point, because the pairing residual interaction is not able to scatter Cooper pairs across the magic shell gaps [44]. We consider that the fragments are pre-formed at this point and experience a nonadiabatic evolution from $R_{0} \simeq 10.5 \mathrm{fm}$ onward, corresponding to the vanishing of all pairing energies.

Consistent with the adiabatic phase, the nonadiabatic evolution is computed at the mean-field level with the TDHF theory [27]. The latter has been widely used to investigate lowenergy nuclear dynamics (see Refs. [45-47] for reviews). Although one-body dissipation mechanisms are well accounted for in the TDHF approach, it does not include the LandauZener effect which is crucial to properly describe dissipation when single-particle levels with different occupation numbers cross. This effect could be partly accounted for with the inclusion of pairing correlations [48] which have been the subject of several recent works [49-52]. Here, the transition between adiabatic and nonadiabatic regimes is supposed to occur after the last crossing. Consequently, the Landau-Zener effect is not expected to affect the dynamics in the nonadiabatic phase.

The TDHF3D code [37] is used with a mesh spacing of $0.8 \mathrm{fm}$ and a time step $1.5 \times 10^{-24} \mathrm{~s}$. The $z=0$ plane represents the 


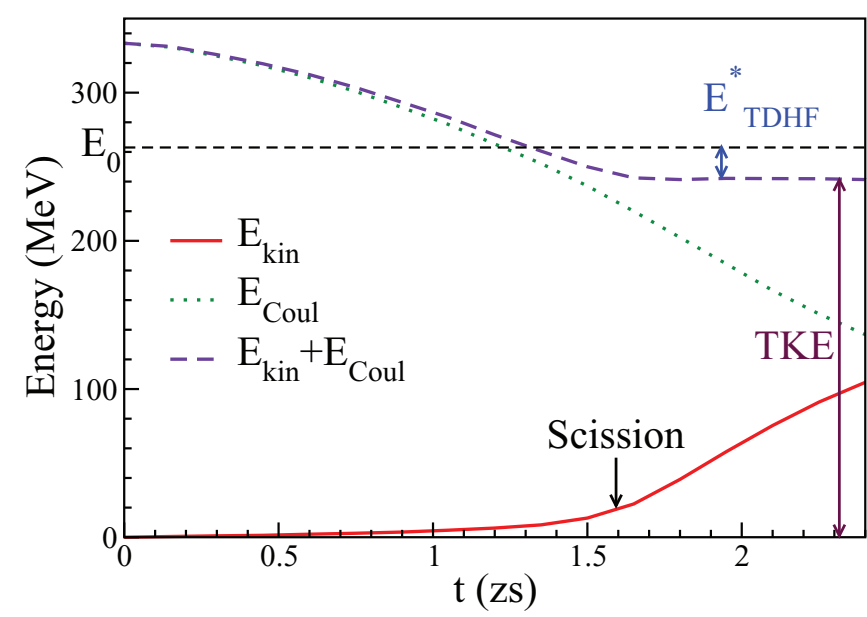

FIG. 3. (Color online) Time evolution of various energies in the nonadiabatic phase (see text).

plane of symmetry. The Cartesian grid extends to $16 \mathrm{fm}$ from the center in $x$ and $y$ and to $64 \mathrm{fm}$ in $z$ direction. Pairing is not included in the dynamics as the fragments maintain their double magicity at all times.

TDHF isodensities are shown in Fig. 1. In this case, the neck remains at elongations up to $R \simeq 15.4 \mathrm{fm}$. It is interesting to quantify the time needed for the nonadiabatic evolution to reach scission. The axis shown in the top of Fig. 1 indicates at which times different values of $R$ are reached. Scission occurs after $\sim 1.6 \mathrm{zs}$ of nonadiabatic evolution. This time is too short for the system to find the minimum of the potential energy surface around scission, which is why the scission point is found to be different when nonadiabatic effects are included.

We now investigate the effect of the nonadiabatic evolution on the kinetic energy $E_{\text {kin }}$ of the fragments. Figure 3 shows the evolution of $E_{\text {kin }}$ and of the potential energy $E_{\text {Coul }}$ arising from mutual Coulomb repulsion. Note that before the fragments are well separated, these energies could depend on the definition of the fragments and on localization effects [12]. When the neck breaks at $t \simeq 1.6 \mathrm{zs}$, the fragments have already acquired a kinetic energy $E_{\text {kin }} \simeq 19 \mathrm{MeV}$. This nonadiabatic contribution to the kinetic energy is usually neglected in models based on the adiabatic approximation [12]. The total kinetic energy (TKE) corresponding to the asymptotic value of $E_{\text {kin }}$ can be obtained by summing the Coulomb and kinetic energies when the nuclear attraction between the fragments vanishes. We get from Fig. 3 a TKE of $241 \mathrm{MeV}$. This TKE is much larger than the prediction from the Viola systematics [53] which is $\sim 192 \mathrm{MeV}$. This effect, already observed in lighter fermium isotopes [42], can be attributed to the strong spherical shell effects in the fragments which are responsible for the compact shape at scission [43].

A similar analysis can be performed for the excitation energy of the fragments. If we consider spontaneous fission, the ${ }^{264} \mathrm{Fm}$ is initially in its ground state, corresponding to the first potential well at $R \simeq 6.8 \mathrm{fm}$ with an energy $E_{\text {g.s. }} \simeq 275 \mathrm{MeV}$ in Fig. 1. At $R_{0} \simeq 10.5 \mathrm{fm}$, where the transition between adiabatic and nonadiabatic regimes occurs, the potential is at $E_{0} \simeq 263 \mathrm{MeV}$ and the system has acquired an excitation energy during its adiabatic evolution $E_{\text {adiab }}^{*}=E_{\text {g.s. }}-E_{0} \simeq$ $12 \mathrm{MeV}$. Note that $E_{\text {adiab }}^{*}$ is much larger than the pairing energy and our conclusions are not affected by the choice of the pairing strength. During the nonadiabatic evolution, and up to scission, the excitation energy keeps increasing due to dissipation mechanisms. The TDHF approach incorporates the one-body dissipation mechanisms which are dominant at low energy [54,55]. As a result, the TDHF prediction of the excitation energy acquired during the nonadiabatic phase is $E_{\mathrm{TDHF}}^{*}=E_{0}-\mathrm{TKE} \simeq 22 \mathrm{MeV}$. The asymptotic value of the total excitation energy is then $E^{*}=E_{\mathrm{adiab}}^{*}+E_{\mathrm{TDHF}}^{*} \simeq 34 \mathrm{MeV}$. The nonadiabatic evolution is then responsible for almost $\sim 2 / 3$ of the final excitation energy of the fragments.

Further inquiry is required to get a deeper insight into the nature of the excitation energy acquired during the nonadiabatic evolution. In the present case, the magic gap of ${ }^{132} \mathrm{Sn}$ is expected to hinder incoherent particle-hole excitations and subsequent thermalization of the fragments. However, collective vibrations, some of which lie at low excitation energies, could be easily excited. Such collective modes are accounted for in the TDHF framework [54,56]. The TDHF simulation of the post-scission evolution of the fragments can then be used to investigate the excitation of such vibrations.

Figures 4(a)-4(d) show the evolution of different multipole moments computed for $z>0$ up to a time $T=5.7 \mathrm{zs}$ before the fragments reach the edge of the grid. At $t \geqslant t_{1} \simeq 1.8 \mathrm{zs}$, i.e., after the neck has fully disappeared, these moments exhibit oscillations associated with vibrational modes of the outgoing fission fragments. The Fourier transform of $\left[Q(t)-Q\left(t_{1}\right)\right]$ $f\left(t-t_{1}\right)$, where $t \geqslant t_{1}$ and $f(t)=\cos ^{2}\left[\pi t / 2\left(T-t_{1}\right)\right]$ is a filtering function to avoid spurious oscillations in the Fourier analysis, are shown in Figs. 4(e)-4(h) (solid lines). They are compared with the same quantities computed after a boost $e^{-i k \hat{Q}}$ applied to the ${ }^{132} \mathrm{Sn}$ HF ground state with a boost velocity, $k$, small enough to be in the linear regime, i.e., to have $Q(t) \propto k$ (dashed lines). This provides a numerical estimate of the random-phase approximation (RPA) strength function of $\hat{Q}$ [44].

The higher energy peaks in the strength functions are associated with giant resonances. Apart from the high-energy octupole resonance (HEOR), all giant resonances are excited in the fission fragments. However, the excitation of low-lying collective vibrations is predominant for the octupole ( $3_{1}^{-}$state) and quadrupole $\left(2_{1}^{+}\right.$state) modes. Such vibrations are often excited in fusion reactions [57], as shown in recent TDHF calculations [58,59]. The isovector dipole response and, to a lesser extent, the monopole one also exhibit other high-energy modes not visible in the RPA strength functions. A possible explanation is that these vibrations are built on top of a static polarization induced by the Coulomb interaction with the other fragment. Indeed, the isovector dipole moment, which is proportional to the distance between the proton and neutron centers of mass, is almost always positive [see Fig. 4(d)]. Note that couplings between collective modes in large amplitude motion [60] could also induce nonlinearities in the vibrational spectra [61,62].

Finally, to test this approach with experimental data, similar calculations have been performed for ${ }^{258} \mathrm{Fm}$. In this case, neutron pairing does not vanish. The TDHF calculations 

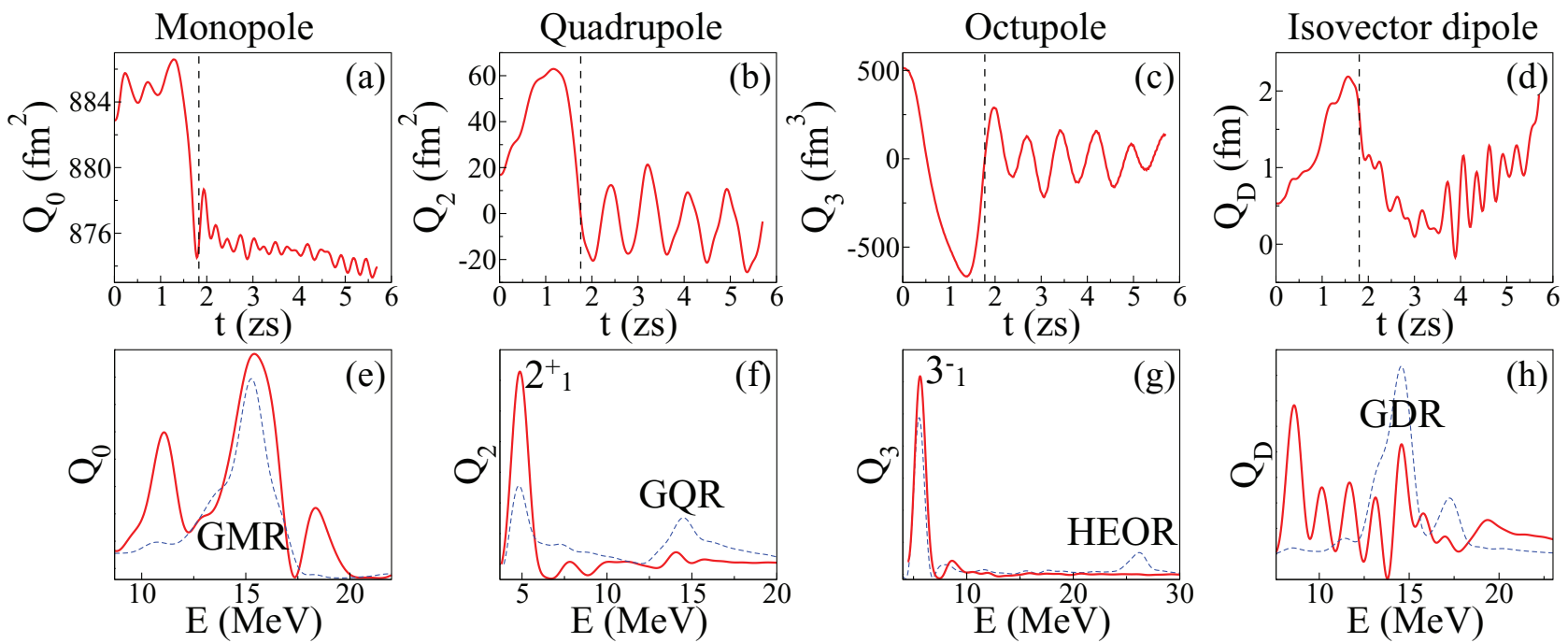

FIG. 4. (Color online) (a)-(d) Evolution of multipole moments in the nonadiabatic phase. (e)-(h) Fourier transforms are computed for $t>t_{1}=1.8 \mathrm{zs}$ (right of dashed line in top panels). The RPA strength functions of ${ }^{132} \mathrm{Sn}$ are plotted with dashed lines (bottom) with arbitrary normalization.

are performed with frozen occupation numbers starting at $R_{0}=12-13 \mathrm{fm}$, for which the occupation numbers are close to the post-scission ones. The calculated TKEs are in the range $238-241 \mathrm{MeV}$, in relatively good agreement with the high-energy mode in ${ }^{258} \mathrm{Fm}(\mathrm{TKE} \sim 232 \mathrm{MeV})$ [42].

Symmetric fission of ${ }^{258,264} \mathrm{Fm}$ has been studied. For the first time, adiabatic and nonadiabatic phases of fission are described with realistic mean-field codes. The evolution is assumed to be adiabatic until the fragments' identity can be established from their shell structure. Nonadiabatic effects are then investigated employing the TDHF evolution toward scission. This nonadiabatic evolution affects the kinetic and excitation energies of the fragments. The post-scission TDHF evolution of the fragments is also used to analyze their vibrational modes. As in the case of fusion, the low-lying collective vibrations are more easily excited than giant resonances. The present techniques could be easily extended to other systems, including asymmetric fission, and other observables, such as neutron emission [4]. Recent mean-field codes [50-52,63] including pairing could be used. Extensions of these codes to compute mass and charge distributions of the fragments could also be used $[64,65]$ in order to compare with experimental data [66].

P. Quentin, L. Bonneau, D. J. Hinde, M. Dasgupta, and E. Williams are thanked for useful comments and discussions. This work has been supported by the Australian Research Council Grant No. FT120100760 and by the US Department of Energy Grant No. DE-FG02-96ER40975 with Vanderbilt University. The calculations have been performed on the NCI National Facility in Canberra, Australia, which is supported by the Australian Commonwealth Government.
[1] O. Hahn and F. Strassmann, Naturwissenschaften 27, 11 (1939).

[2] L. Meitner and O. R. Frisch, Nature 143, 239 (1939).

[3] S. G. Frauendorf and C. Guet, Ann. Rev. Nucl. Part. Sci. 51, 219 (2001).

[4] D. J. Hinde, D. Hilscher, H. Rossner, B. Gebauer, M. Lehmann, and M. Wilpert, Phys. Rev. C 45, 1229 (1992).

[5] J. Moreau and K. Heyde, in The Nuclear Fission Process, edited by C. Wagemans (CRC, Boca Raton, FL, 1991).

[6] M. Rizea and N. Carjan, Nucl. Phys. A 909, 50 (2013).

[7] N. Dubray, H. Goutte, and J.-P. Delaroche, Phys. Rev. C 77, 014310 (2008).

[8] H. Goutte, J. F. Berger, P. Casoli, and D. Gogny, Phys. Rev. C 71, 024316 (2005).

[9] L. Bonneau, Phys. Rev. C 74, 014301 (2006).

[10] A. Staszczak, A. Baran, J. Dobaczewski, and W. Nazarewicz, Phys. Rev. C 80, 014309 (2009).
[11] J. C. Pei, W. Nazarewicz, J. A. Sheikh, and A. K. Kerman, Phys. Rev. Lett. 102, 192501 (2009).

[12] W. Younes and D. Gogny, Phys. Rev. Lett. 107, 132501 (2011).

[13] M. Warda and J. L. Egido, Phys. Rev. C 86, 014322 (2012).

[14] H. Abusara, A. V. Afanasjev, and P. Ring, Phys. Rev. C 85, 024314 (2012).

[15] M. Mirea, Phys. Lett. B 717, 252 (2012).

[16] A. Staszczak, A. Baran, and W. Nazarewicz, Phys. Rev. C 87, 024320 (2013).

[17] J. D. McDonnell, W. Nazarewicz, and J. A. Sheikh, Phys. Rev. C 87, 054327 (2013).

[18] J. Sadhukhan, K. Mazurek, A. Baran, J. Dobaczewski, W. Nazarewicz, and J. A. Sheikh, Phys. Rev. C 88, 064314 (2013).

[19] N. Schunck, D. Duke, H. Carr, and A. Knoll, arXiv:1311.2616.

[20] N. Schunck, D. Duke, and H. Carr, arXiv:1311.2620. 
[21] M. Baranger and M. Vénéroni, Ann. Phys. (NY) 114, 123 (1978).

[22] P. Möller, D. G. Madland, A. J. Sierk, and A. Iwamoto, Nature 409, 785 (2001).

[23] J. Randrup and P. Möller, Phys. Rev. Lett. 106, 132503 (2011).

[24] B. D. Wilkins, E. P. Steinberg, and R. R. Chasman, Phys. Rev. C 14, 1832 (1976).

[25] R. Bernard, H. Goutte, D. Gogny, and W. Younes, Phys. Rev. C 84, 044308 (2011).

[26] A. S. Umar, V. E. Oberacker, J. A. Maruhn, and P.-G. Reinhard, J. Phys. G 37, 064037 (2010).

[27] P. A. M. Dirac, Math. Proc. Camb. Phil. Soc. 26, 376 (1930).

[28] J. W. Negele, S. E. Koonin, P. Möller, J. R. Nix, and A. J. Sierk, Phys. Rev. C 17, 1098 (1978).

[29] P. Möller and A. Iwamoto, Phys. Rev. C 61, 047602 (2000).

[30] R. Gherghescu, D. Poenaru, and W. Greiner, Z. Phys. A 354, 367 (1996).

[31] T. Asano, T. Wada, M. Ohta, T. Ichikawa, S. Yamaji, and H. Nakahara, J. Nucl. Radiochem. Sci. 5, 1 (2004).

[32] J. Dvorak, M. Block, C. Düllmann, S. Heinz, R.-D. Herzberg, and M. Schädel, Nucl. Instrum. Methods Phys. Res., Sect. A 652, 687 (2011).

[33] V. I. Zagrebaev, Y. T. Oganessian, M. G. Itkis, and W. Greiner, Phys. Rev. C 73, 031602 (2006).

[34] C. Golabek and C. Simenel, Phys. Rev. Lett. 103, 042701 (2009).

[35] D. J. Kedziora and C. Simenel, Phys. Rev. C 81, 044613 (2010).

[36] P. Bonche, H. Flocard, and P. H. Heenen, Comput. Phys. Commun. 171, 49 (2005).

[37] K.-H. Kim, T. Otsuka, and P. Bonche, J. Phys. G 23, 1267 (1997).

[38] T. Skyrme, Phil. Mag. 1, 1043 (1956).

[39] M. Bender, P.-H. Heenen, and P.-G. Reinhard, Rev. Mod. Phys. 75, 121 (2003).

[40] P. Möller, A. J. Sierk, T. Ichikawa, A. Iwamoto, R. Bengtsson, H. Uhrenholt, and S. Åberg, Phys. Rev. C 79, 064304 (2009).

[41] M. Kowal, P. Jachimowicz, and A. Sobiczewski, Phys. Rev. C 82, 014303 (2010).

[42] E. K. Hulet, J. F. Wild, R. J. Dougan, R. W. Lougheed, J. H. Landrum, A. D. Dougan, M. Schadel, R. L. Hahn, P. A. Baisden, C. M. Henderson, R. J. Dupzyk, K. Sümmerer, and G. R. Bethune, Phys. Rev. Lett. 56, 313 (1986).

[43] T. Ichikawa, A. Iwamoto, and P. Möller, Phys. Rev. C 79, 014305 (2009).
[44] P. Ring and P. Schuck, The Nuclear Many-Body Problem (Springer-Verlag, New York, 1980).

[45] J. W. Negele, Rev. Mod. Phys. 54, 913 (1982).

[46] C. Simenel, Eur. Phys. J. A 48, 152 (2012).

[47] C. Simenel, in Clusters in Nuclei, Vol. 3, edited by C. Beck, Lecture Notes in Physics, Vol. 875 (Springer, New York, 2014), p. 95 .

[48] J. Błocki and H. Flocard, Nucl. Phys. A 273, 45 (1976).

[49] B. Avez, C. Simenel, and P. Chomaz, Phys. Rev. C 78, 044318 (2008)

[50] S. Ebata, T. Nakatsukasa, T. Inakura, K. Yoshida, Y. Hashimoto, and K. Yabana, Phys. Rev. C 82, 034306 (2010).

[51] I. Stetcu, A. Bulgac, P. Magierski, and K. J. Roche, Phys. Rev. C 84, 051309 (2011).

[52] G. Scamps and D. Lacroix, Phys. Rev. C 87, 014605 (2013).

[53] V. E. Viola, K. Kwiatkowski, and M. Walker, Phys. Rev. C 31, 1550 (1985).

[54] P. Bonche, S. Koonin, and J. W. Negele, Phys. Rev. C 13, 1226 (1976).

[55] S. E. Koonin, K. T. R. Davies, V. Maruhn-Rezwani, H. Feldmeier, S. J. Krieger, and J. W. Negele, Phys. Rev. C 15, 1359 (1977).

[56] J. Błocki and H. Flocard, Phys. Lett. B 85, 163 (1979).

[57] M. Dasgupta, D. J. Hinde, N. Rowley, and A. M. Stefanini, Ann. Rev. Nucl. Part. Sci. 48, 401 (1998).

[58] C. Simenel, R. Keser, A. S. Umar, and V. E. Oberacker, Phys. Rev. C 88, 024617 (2013).

[59] C. Simenel, M. Dasgupta, D. J. Hinde, and E. Williams, Phys. Rev. C 88, 064604 (2013).

[60] C. Simenel, P. Chomaz, and G. de France, Phys. Rev. Lett. 86, 2971 (2001).

[61] C. Simenel and P. Chomaz, Phys. Rev. C 68, 024302 (2003).

[62] C. Simenel and P. Chomaz, Phys. Rev. C 80, 064309 (2009).

[63] J. A. Maruhn, P.-G. Reinhard, P. D. Stevenson, and A. S. Umar, arXiv: 1310.5946.

[64] C. Simenel, Phys. Rev. Lett. 105, 192701 (2010).

[65] C. Simenel, Phys. Rev. Lett. 106, 112502 (2011).

[66] K.-H. Schmidt, S. Steinhäuser, C. Bökstiegel, A. Grewe, A. Heinz, A. Junghans, J. Benlliure, H.-G. Clerc, M. de Jong, J. Müller, M. Pfützner, and B. Voss, Nucl. Phys. A 665, 221 (2000). 